\title{
Die Herztätigkeit von Heringsembryonen bei verschiedenen Temperaturen ${ }^{1}$
}

\author{
Harald RosentHaL \\ Biologische Anstalt Helgoland, Zentrale, Hamburg 50
}

\begin{abstract}
The heart activity of herring embryos at different temperatures. In artificially spawned herring eggs (Kiel), embryonic heart activity starts at $8.0^{\circ} \pm 0.2^{\circ} \mathrm{C}$ water temperature, slowly and irregularly at the 5 th day after fertilizarion. On the 6th day an average of about 17 beats per minute $( \pm 10$ to $40 \%$; maximal $\pm 70 \%$ ) is readhed. 16 to 18 days after fertilization, heart frequency reaches average values between 58 and 66 beats per minute; regression line: $y=39.9+0.17(x-274.7)$. When the temperature was kept at $10^{\circ} \pm 0.1^{\circ} \mathrm{C}$ during the whole incubation period, the heart began to contract rhythmically at the end of the 4 th day after fertilization performing 20 to 21 beats per minute; on the 12th day of incubation, heart activity increased to about 56 beats per minute following the regression line y $=40.5+0.20$ (x-215.4). A significant decrease in variability during development could not be observed. After the 8th day of incubation, increasing water temperature (from $10^{\circ}$ to $14^{\circ} \mathrm{C}$ ) causes considerable undulations of the heart activity curve, following an initial overshoot response.
\end{abstract}

\section{EINLEITUNG}

Die Herztätigkeit der Fische ist in zahlreichen Studien untersucht worden, vor allem hinsichtlich des Einflusses der Temperatur auf die Pulsationsfrequenz bei adulten Individuen (KoEHNLEIN 1933; MARKOWSKY 1933). Nur wenige Untersuchungen befassen sich mit dem Frequenzgang der Herztätigkeit während der Embryonalentwicklung und dessen Temperaturabhängigkeit (Huggel 1959). Die Herztätigkeit nimmt im Laufe der Entwicklung ständig zu. Diese Tatsache ist erstmals von ANDERsen (1930) an Embryonen von Salmo fario nachgewiesen worden. Ferner ist bekannt, daß bei zahlreichen Fischen die Herztätigkeit der Frühstadien durch beträchtliche individuelle Frequenzdifferenzen gekennzeichnet ist, während die Spätstadien eine strengere Rhythmizität aufweisen.

Die vorliegende Arbeit befaßt sich mit der Herzfrequenz des Herings (Clupea barengus) während der Embryonalentwicklung bei zwei konstanten Temperaturen, $8^{0}$ und $10^{\circ} \mathrm{C}$, sowie mit den Veränderungen der Herztätigkeit bei plötzlicher Temperaturerhöhung von $10^{\circ}$ auf $14^{\circ} \mathrm{C}$ kurz vor dem Augenpunktstadium.

1 Herrn Professor Dr. FrIEDRICH KrÜGER anläßlich seines 65. Geburtstags am 18. August 1967 in Verehrung gewidmet. 


\section{MATERIAL UND METHODEN}

Die Erbrütungen erfolgten an Kieler Frühjahrslaichern und an Downsheringen. Die künstliche Befruchtung wurde unmittelbar nach dem Fang (Downs: $30 \% \mathrm{~S} ; 10^{\circ}$ bis $11^{\circ} \mathrm{C}$ ) oder 1 bis 2 Stunden später (Kiel: $15 \%$; $; 8^{\circ} \mathrm{C}$ ) vorgenommen.

Während des Transports traten geringfügige Temperaturerhöhungen ein (Downs: 0,5 bis $1,0^{\circ} \mathrm{C}$; Kiel: 0,5 bis $0,8^{\circ} \mathrm{C}$ ). Die Transportzeiten rom Fangplatz zum Labor betrugen 2 bis 3 Stunden (Kiel) und 60 Stunden (Downs). Anschließend wurden die Eier der Kieler Frühjahrslaicher in Hälterungsgefäße überführt, die in einem Temperierbad während der Dauer der Erbrütungsversuche auf $8,0^{\circ} \pm 0,2^{\circ} \mathrm{C}$ gehalten wurden; methodische Einzelheiten bei Paffenhöfer \& Rosenthal (1967). Die Erbritungsversuche an den Downsheringen wurden in einem dunklen temperaturkonstanten Raum $\left(10^{\circ} \pm 0,1^{\circ} \mathrm{C}\right)$ durchgeführt.

Der Herzschlag wurde unter dem Mikroskop beobachtet und die Pulsfrequenz mit der Stoppuhr (Genauigkeit $\pm 0,1 \mathrm{sec}$ ) ermittelt. Gemessen wurde jeweils die Zeit, in der das Herz $25 \mathrm{mal}$ schlägt. An einem Individuum wurden stets 3 aufeinanderfolgende Messungen durchgeführt und der erhaltene Mittelwert auf Frequenz pro Minute umgerechnet.

\section{ERGEBNISSE}

Bei einer Erbrïtungstemperatur von $8^{\circ} \mathrm{C}$ setzt die Tätigkeit des embryonalen Herzschlauchs mit langsamen, unregelmäßigen Pulsationen am 5. Tag nach der Befruchtung ein. Die Region der Pulsbildung liegt im ersten Viertel des Herzschlauchs. Die Pulsationen laufen wellenförmig über eine zunächst nur kurze Strecke in arterieller Richtung und breiten sich im Laufe der weiteren Embryonalentwicklung über den gesamten Herzschlauch aus. Die Kontraktionen sind sehr unregelmäßig. Die Pulsationsfrequenz beträgt kurz nach Beginn der Herztätigkeit (6. Tag nach der Befruchtung) im Mittel 17 bis 18 Schläge pro Minute. Die Unregelmäßigkeiten der Pulsationen können zeitweise Abweichungen bis über $70 \%$ von der mittleren Frequenz erreichen; zeitweise sind sie gering. Eine strengere Rhythmizität der Spätstadien konnte nur gelegentlich beobachtet werden. Auch hier treten oftmals starke Abweichungen auf. Einige Beispiele sind in Tabelle 1 wiedergegeben. Bei $8^{\circ} \mathrm{C}$ steigt die Herzfrequenz nach Pulsationsbeginn von 17 bis 18 (6. Inkubationstag) auf 58 bis 66 (16. bis 18. Inkubationstag) Schläge pro Minute. Die aus 587 Einzelmessungen berechnete Regression folgt der Gleichung $y=39,9+0,17(x-274,7)(A b b .1)$, wobei $x$ das Alter des Embryos in Stunden nach der Befruchtung angibt.

Bei $10^{\circ} \mathrm{C}$ ist der Pulsbeginn bereits gegen Ende des 4. Tages nach der Befruchtung zu beobachten. Um größere individuelle Frequenzdifferenzen - wie sie beim $8^{0}-\mathrm{C}-\mathrm{Ver}-$ such auftraten $-z u$ vermeiden, wurden hier die Messungen während der gesamten Inkubationszeit stets an den gleichen Individuen vorgenommen. Generell ist die Pulsfrequenz zu Beginn der Kontraktionen des Herzschlauchs mit 20 bis 21 Schlägen pro Minute um etwa $22 \%$ höher als bei $8^{\circ} \mathrm{C}$. Die Herzschlagfrequenz steigt während der Entwicklung entsprechend der kürzeren Inkubationszeit bei $10^{\circ} \mathrm{C}$ auch rascher an. Sie erreicht am 12. Tag nach der Befruchtung 55 bis 57 Schläge pro Minute. Dieser Wert 


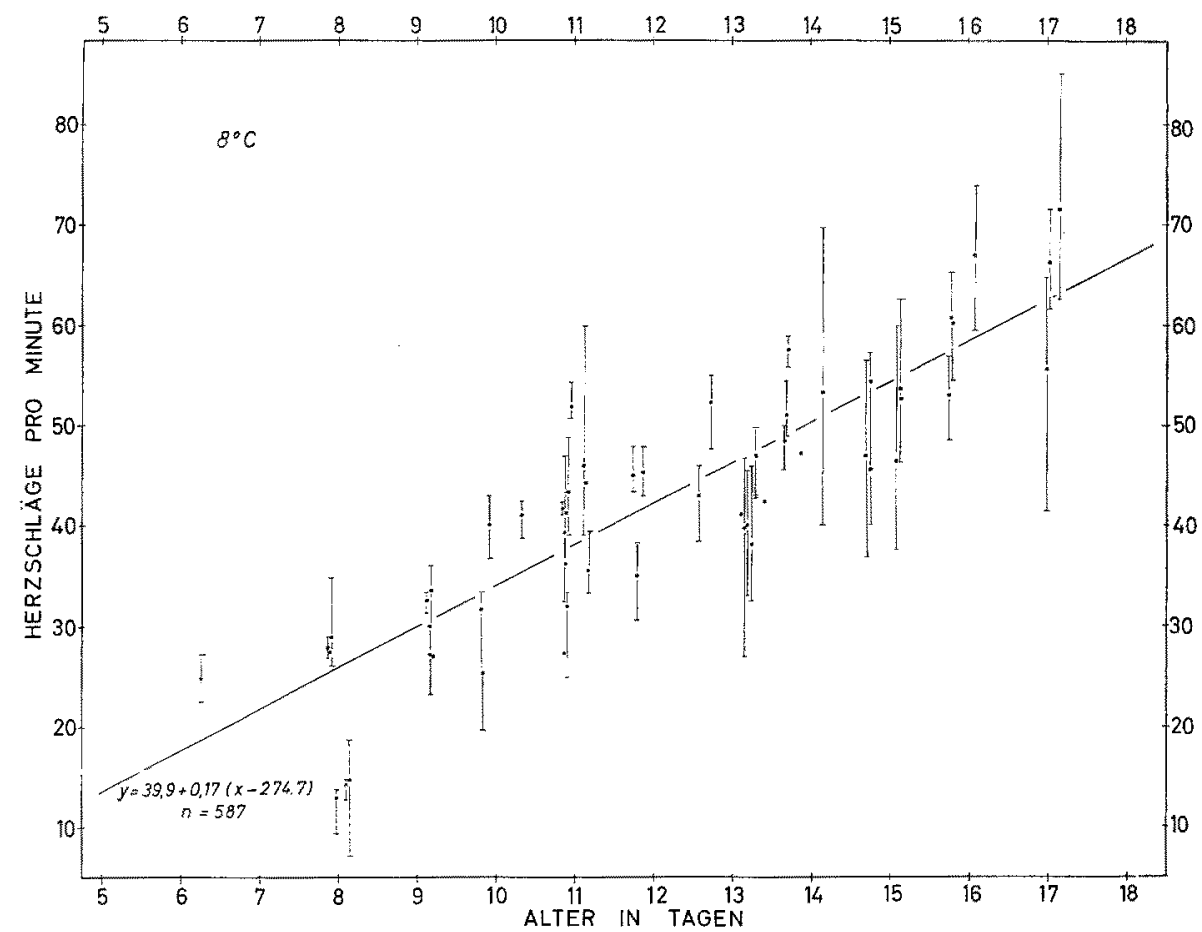

Abb. 1: Die Herztätigkeit der Embryonen von Clmpea barengus L. (Kieler Frühjahrslaicher) bei $8,0^{\circ} \pm 0,2^{\circ} \mathrm{C}$ in Abhängigkeit vom Alter (Tage nach der Befruchtung). Mittelwerte aus jeweils 10 bis 15 Einzelmessungen. Die vertikalen Linien geben die während einer Meßreihe beobachtete Variationsbreite an. Streuung $s=6,5$

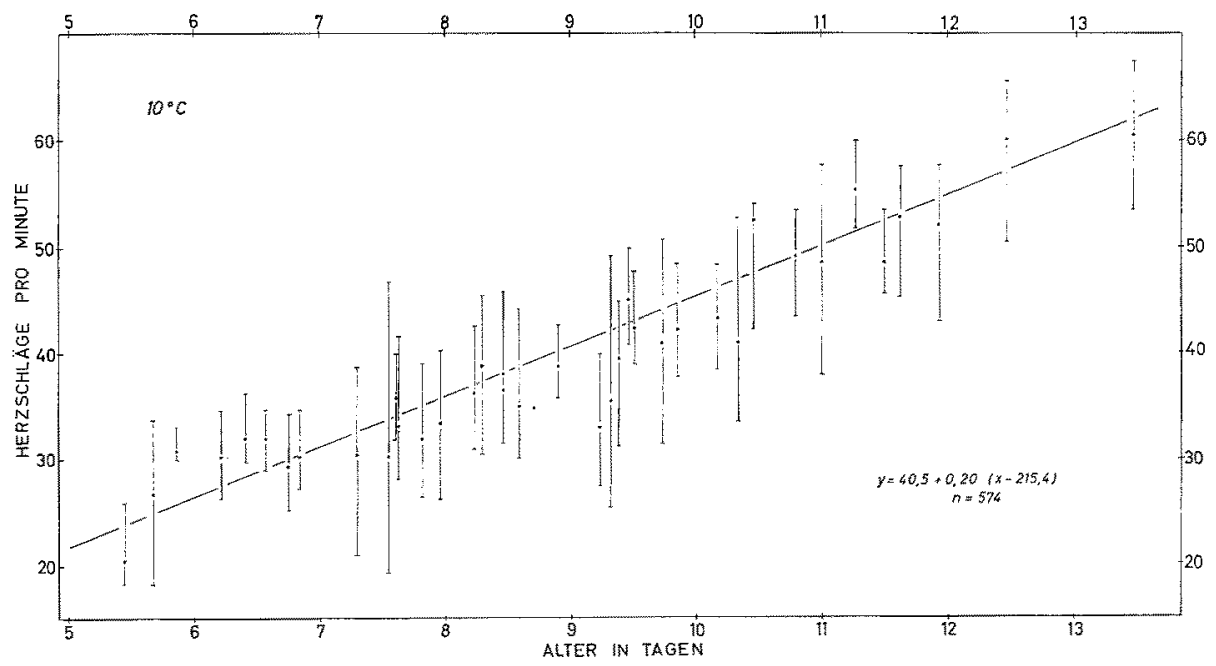

Abb. 2: Die Herztätigkeit der Embryonen des Herings (Downs-Eier) bei $10^{\circ} \pm 0,1^{0} \mathrm{C}$ in $\mathrm{Ab}$ hängigkeit vom Alter (Tage nach der Befruchtung). Mittelwerte aus jeweils 12 bis 30 Einzelmessungen. Die Vertikalen geben die während einer Meßreihe beobachtete Variationsbreite an. Streuung $s=4,9$ 
Tabelle 1

Mittlere Herzschlagfrequenz bei verschieden alten Embryonen des Herings (Clupea harengus). Erbrütungstemperatur $8,0^{\circ} \pm 0,2^{\circ} \mathrm{C}$. Durchschnittswerte aus jeweils 15 Einzelmessungen

\begin{tabular}{|ccccc|}
\hline $\begin{array}{c}\text { Alter in Tagen } \\
\text { nach der } \\
\text { Befruchtung }\end{array}$ & $\begin{array}{c}\text { Mittlere } \\
\text { Frequenz } \\
\text { pro Minute }\end{array}$ & $\begin{array}{c}\text { Maximale Abweichungen } \\
\text { von der mittleren Frequenz } \\
+\end{array}$ & $\begin{array}{c}\text { Variations- } \\
\text { breite } \\
(0 / 0)\end{array}$ \\
\hline 6 & 25,2 & 2,2 & 2,7 & 19,4 \\
7 & 28,0 & 1,0 & 0,9 & 6,8 \\
7 & 29,0 & 5,9 & 3,1 & 31,0 \\
8 & 14,7 & 4,1 & 7,6 & 79,6 \\
9 & 25,4 & 2,9 & 5,8 & 34,2 \\
9 & 31,8 & 1,7 & 1,5 & 10,1 \\
10 & 36,2 & 4,6 & 6,1 & 29,6 \\
10 & 41,0 & 1,5 & 2,5 & 9,7 \\
11 & 44,4 & 7,8 & 4,1 & 26,8 \\
11 & 45,3 & 1,5 & 2,1 & 17,9 \\
12 & 43,1 & 3,0 & 4,6 & 11,8 \\
12 & 52,4 & 2,6 & 3,6 & 8,7 \\
13 & 48,4 & 1,6 & 2,6 & 30,6 \\
13 & 40,2 & 5,6 & 6,7 & 34,0 \\
14 & 45,5 & 9,1 & 6,1 & 13,9 \\
14 & 53,9 & 3,2 & 4,3 & 54,4 \\
15 & 46,5 & 14,1 & 11,2 & 34,1 \\
15 & 53,6 & 10,9 & 7,4 & 17,4 \\
15 & 60,8 & 4,4 & 6,2 & 12,3 \\
15 & 60,4 & 4,1 & 3,3 & 19,8 \\
16 & 55,7 & 9,0 & 14,3 & 14,5 \\
16 & 67,0 & 5,8 & 7,5 & 4,8 \\
17 & 66,3 & 4,8 & & \\
\hline
\end{tabular}

wird bei $8^{\circ} \mathrm{C}$ erst am 15. bis 16. Inkubationstag erreicht. Die aus 574 Einzelmessungen errechnete Regression lautet: $y=40,5+0,20$ ( $x-215,4$ ) (Abb. 2).

Uberführt man 8 Tage alte Embryonen innerhalb von 12 Stunden von $10^{\circ}$ auf $14^{0} \mathrm{C}$, so steigt die Pulsfrequenz zunächst erheblich an. Bald jedoch beginnt der Herzschlag zu undulieren. Diese Tendenz zeigt sich, wenn man die Mittelwerte der einzel-

Tabelle 2

Mittlere Herzschlagfrequenz bei verschieden alten Embryonen des Herings (Clupea harengus). Erbrütungstemperatur $10,0^{\circ} \pm 0,1^{\circ} \mathrm{C}$. Durchschnittswerte aus jeweils 15 Einzelmessungen

\begin{tabular}{|ccccc|}
\hline $\begin{array}{c}\text { Alter in Tagen } \\
\text { nach der } \\
\text { Befruchtung }\end{array}$ & $\begin{array}{c}\text { Mittlere } \\
\text { Frequenz } \\
\text { pro Minute }\end{array}$ & $\begin{array}{c}\text { Maximale Abweichungen } \\
\text { von der mittleren Frequenz } \\
+\end{array}$ & $\begin{array}{c}\text { Variations- } \\
\text { breite } \\
(\mathbf{0} / 0)\end{array}$ \\
\hline 5 & 20,6 & 5,4 & 2,3 & 36,8 \\
6 & 30,2 & 4,4 & 4,0 & 28,1 \\
7 & 35,8 & 4,2 & 3,9 & 22,6 \\
8 & 38,9 & 6,6 & 8,4 & 38,3 \\
9 & 45,0 & 5,0 & 4,2 & 20,5 \\
10 & 52,7 & 1,3 & 10,4 & 22,4 \\
11 & 55,5 & 4,5 & 3,8 & 14,9 \\
11 & 48,6 & 5,0 & 3,1 & 16,7 \\
12 & 60,6 & 6,8 & 7,0 & 22,9 \\
\hline
\end{tabular}


nen Meßreihen unter Berücksichtigung der Beobachtungsintervalle betrachtet. In Abbildung 3 sind außerdem die Meßergebnisse für 5 Individuen getrennt aufgetragen (jeweils Mittelwerte aus 3 Messungen). Deutlich fallen die individuellen Frequenzdifferenzen $z$ wischen den einzelnen Individuen auf. Während die Undulationen der Herzfrequenz beim Individuum 2 die größten Schwankungen erreichen, stellen sie sich beim Individuum 5 auf ein höheres Niveau ein. Die Embryonen 1 bis 4 starben noch im Ei; Embryo 5 starb kurz nach dem Schlüpfen. Diejenigen Embryonen, die den Schlüpftermin unbeschadet erreichten (Individuen Nr. 6 bis 10; Abb. 3), hatten am 12. Tag nach der Befruchtung im Mittel eine Pulsfrequenz von über 70 Schlägen erreicht. In diesem Versuch schlüpften nur wenige Individuen.

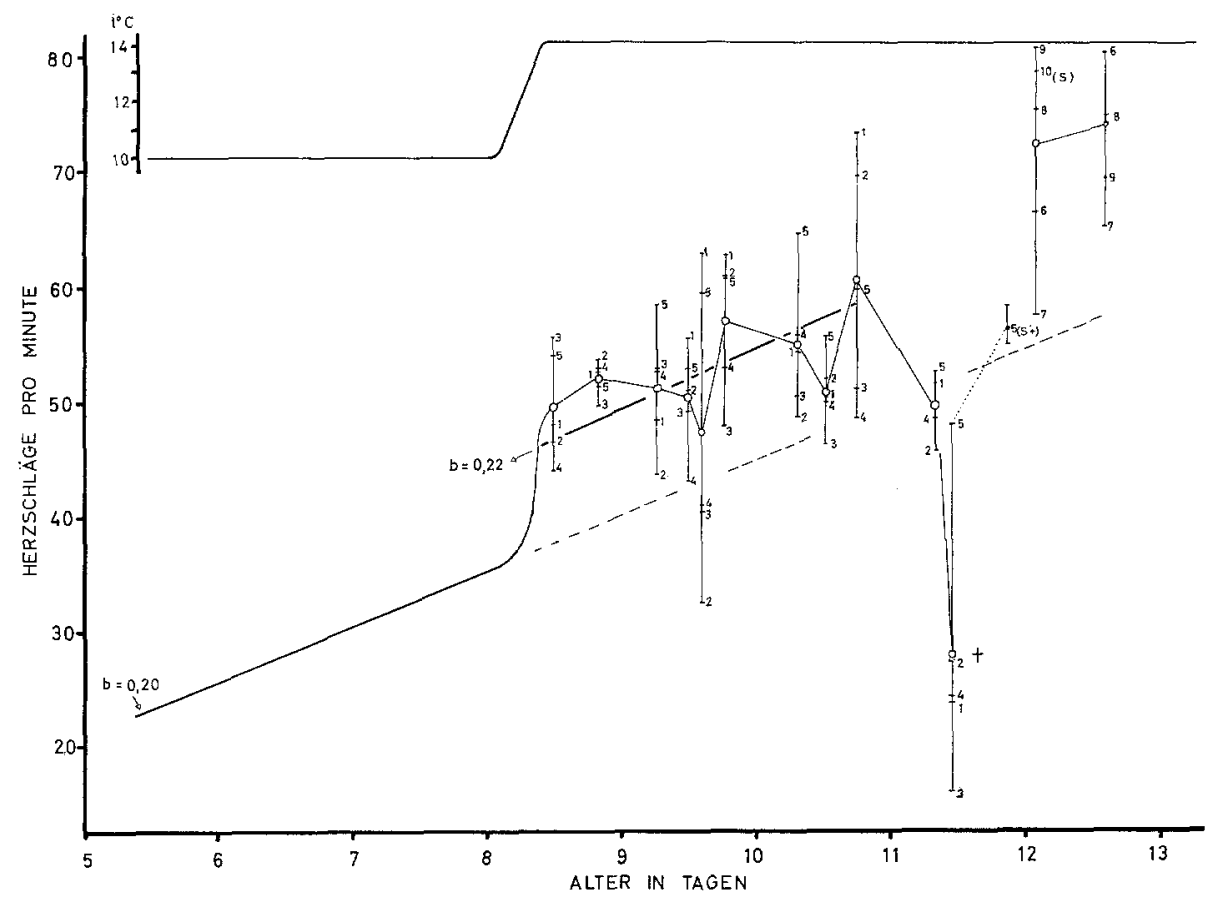

Abb. 3: Reaktion der Herztätigkeit der Embryonen des Herings auf eine plötzliche Temperaturänderung nach dem 8 . Tage der Inkubation von $10^{\circ}$ auf $14^{\circ} \mathrm{C}$. Die Mitrelwerte aus jeweils 3 Messungen sind für jedes beobachtete Individuum (Nr. 1 bis $5 ; 6$ bis 10) getrennt aufgetragen. Die Kreise kennzeichnen die Mittelwerte aus allen Messungen an den 5 Individuen

\section{DISKUSSION}

Bei Heringsembryonen nehmen die Herzpulsationen pro Minute während der Entwicklung stetig zu. Dabei treten starke individuelle Unterschiede auf. Eine Deutungsmöglichkeit für diese Erscheinung geben die differenzierteren Befunde von HUGGEL (1959). Huggel konnte an Embryonen von Salmo fario zeigen, daß sich die Automatie während der Entwicklung in mehreren Teilprozessen ausbreitet. Der Ausbreitung der 
primären Automatiezone in venöser und arterieller Richtung mit deutlichem Frequenzgefälle folgt die Ausbildung von getrennten Automatiezonen mit maximaler Herzfrequenz. Diese Zonen entsprechen den embryonalen Herzzentren. Die Funktion der einzelnen Herzabschnitte wird nach Pulsbeginn noch nicht zentral reguliert. Es kommt zur zeitweisen Antagonie oder Synchronisation der Tätigkeit einzelner Herzabschnitte. Bei Temperaturerhöhung scheint sich die Abweichung der Eigenfrequenz der embryonalen Herzzentren zu erhöhen und auf diese Weise die beobachteten Undulationen zu verursachen (Abb. 3). Solche Undulationen der Herzaktivität konnten an Heringsembryonen unter Stress-Bedingungen beobachtet werden (KINNE \& RosenTHAL 1967). Sie sind auch bei Krebsen nachgewiesen worden, so zum Beispiel bei Gammarus duebeni (KINNE 1952, 1963).

Der geringe Schlüpferfolg nach der Überführung von $10^{\circ}$ auf $14^{\circ} \mathrm{C}$ läßt vermuten, daß diese Temperaturerhöhung vor dem Augenpunktstadium zu hoch war, um schadlos überstanden werden zu können. Wie HaMdorf (1961) an Embryonen von Salmo irideus zeigen konnte, steigt die Thermoresistenz und die mittlere Sterbezeit mit fortschreitender Embryonalentwicklung rasch an. Die Sterberaten werden geringer, die Sterbezeiten länger. Die Embryonen sind nach dem Augenpunktstadium in stärkerem Maße fähig, sich veränderten Umweltbedingungen anzupassen. An der Grenze zwischen letaler und subletaler Schädigung durch Veränderungen in den Umweltbedingungen ( $z$. B. Temperatur) erscheinen Amplitude und Frequenz der Pulsundulationen des embryonalen Herzschlauchs als ein geeignetes Maß zur Ermittlung der physiologischen Reaktionsbreite des Einzelindividuums.

In weiteren Versuchen soll geprüft werden, inwieweit geringfügige Temperaturänderungen und Temperaturschwankungen signifikante Undulationen der Herztätigkeit verursachen können.

\section{ZUSAMMENFASSUNG}

1. Die Herztätigkeit (Anzahl der Pulsationen) der Embryonen des Herings wurde vom Pulsationsbeginn an bis zum Schlïpfen bei $8^{\circ}$ und $10^{\circ} \mathrm{C}$ gemessen.

2. Die Pulsationswerte lassen starke individuelle Unterschiede erkennen.

3. Bei $8^{\circ} \mathrm{C}$ setzt die Herztätigkeit am 5. Tag nach der Befruchtung ein. Sie erreicht am 6. Tag 17 bis 18 Schläge pro Minute und steigt bis zum Schlüpfen (16. bis 18. Tag) auf 58 bis 66 Schläge an. Regression $y=39,9+0,17$ ( $x-274,7$ ) (Abb. 1).

4. Bei $10^{\circ} \mathrm{C}$ kann der Pulsationsbeginn bereits am 4 . Inkubationstag beobachtet werden. Die Herzfrequenz steigt von 20 bis 21 Schläge (5. Tag) auf 55 bis 57 (12. Tag) pro Minute an. Regression $y=40,5+0,20$ ( $x-215,4$ ) (Abb. 2).

5. Uberführt man 8 Tage alte Embryonen direkt von $10^{\circ}$ nach $14^{\circ} \mathrm{C}$, so beginnt die Herztätigkeit nach sprunghaftem Anstieg zu undulieren.

Diese Arbeit wurde mit finanzieller Unterstützung der Deutschen Forschungsgemeinschaft durchgeführt (Ki 41/15). 


\section{ZITIERTE LITERATUR}

ANDERSEN, K., 1930. Abhängigkeit der Herzschlagzahl und der Atembewegungen bei Knodhenfischen von der Keimlingsgröße und der Temperatur. Entwicklungsphysiologische Untersuchungen an Bachforellenkeimlingen. Z. vergl. Physiol. 11, 56-103.

Hamdorf, K., 1961. Die Beeinflussung der Embryonal- und Larvalentwicklung der Regenbogenforelle (Salmo irideus Grвв.) durch die Umweltfaktoren $\mathrm{O}_{2}$-Partialdruck und Temperatur. Z. vergl. Physiol. 44, 523-549.

HUGGEx, H., 1959. Experimentelle Untersuchungen über die Automatie, Temperaturabhängigkeit und Arbeit des embryonalen Fischherzens, unter besonderer Berüdksichtigung der Salmoniden und Scylliorhiniden. Z. vergl. Physiol. 42, 63-102.

Kinne, O., 1952. Zur Biologie und Physiologie von Gammarus duebeni LnLLJ., V.: Untersuchungen über Blutkonzentrationen, Herzfrequenz und Atmung. Kieler Meeresforsch. 9, $134-150$.

- 1963. The effects of temperature and salinity on marine and brackish water animals. I. Temperature. Oceanogr. mar. Biol. Ann. Rev. 1, 301-340.

- \& Rosenthal, H., 1967. Effects of sulfuric water pollutants on fertilization, embryonic development and larvae of the herring, Clupea barengus. Mar. Biol. 1, 65-83.

KoennlerN, H., 1933. Untersuchungen iiber die Herztätigkeit der Fische. VI. Pubbl. Staz. zool. Napoli 13, 129-142.

Markowsky, H., 1933. Uber die Beeinflussung der Herztätigkeit durch die Temperatur. Biologia gen. 9, 231-248.

Paffenhiöfer, G.-A. \& Rosenthal, H., 1967. Trockengewicht und Kaloriengehalt sich entwickelnder Heringseier. Helgoländer wiss. Meeresunters. 16 (im Druck). 